\title{
Experimental investigation towards a periodically pumped single-photon source
}

\author{
C. Bödefeld, ${ }^{1}$ J. Ebbecke, ${ }^{2}$ J. Toivonen, ${ }^{3}$ M. Sopanen, ${ }^{3}$ H. Lipsanen, ${ }^{3}$ and A. Wixforth ${ }^{2}$ \\ ${ }^{1}$ Sektion Physik der Ludwig-Maximilians-Universität and Center for NanoScience (CeNS), Geschwister-Scholl-Platz 1, 80539 Munich, \\ Germany \\ ${ }^{2}$ Institut für Physik der Universität Augsburg, Experimentalphysik I, Universitätsstrasse 1, 86135 Augsburg, Germany \\ ${ }^{3}$ Optoelectronics Laboratory, Helsinki University of Technology, Tietotie 3, FIN-02150 Espoo, Finland
}

(Received 23 March 2006; published 10 July 2006)

Experiments towards a periodically pumped single-photon source are presented. The lateral piezoelectric field of a surface acoustic wave dissociates laser-generated two-dimensional excitons into electrons and holes. These carriers are separated by the wave potential and are transported over macroscopic length scales without recombining. When reaching a stress-induced quantum dot in the quantum well they periodically populate the zero-dimensional states and recombine, emitting single photons periodically in time according to the surface acoustic-wave frequency. We have successfully reduced the number of pumped quantum dots down to 100 and have detected a strong blinking photoluminescence signal. By further reducing the number of quantum dots down to 1 a periodically pumped single photon source could be realized.

DOI: 10.1103/PhysRevB.74.035407

PACS number(s): 73.63.Kv, 78.20.Hp, 78.70.Gq

Single-photon sources (SPS's) are of great interest in the vast growing field of information technology. ${ }^{1}$ As the emitted photons of SPS are antibunched, applications become feasible that would be often impossible to realize with classical light sources. The first important area to mention at this point is quantum cryptography. ${ }^{2}$ To transfer information by coding them in a certain way that no one else is able to understand is topic of actual research and has also been a desire for many centuries. If the information has been transmitted by electromagnetic waves, many photons have been carrying the data so far. Utilizing single-photon sources to transfer coded data (either by the state of polarization or in time packages) a new possibilty of securing information is realizable. Due to the noncloning theorem of a single quantum state, nobody can eavesdrop on a single photon without changing his quantum state or even annihilating it. Therefore the actual receptor of the information will know that the secure transmittance of data has been attacked. That is in principle impossible by classical information transmittance.

There are several technological approaches of realizing SPS's with different grades of success. One of the first realized devices that has shown antibunched signals is the socalled single-photon turnstile device. ${ }^{3}$ Multiple-quantumwell structures and alternating tunneling of electron and holes are used to realize the SPS. The disadvantage of this Coulomb-blockade-controlled mesoscopic $p$ - $n$ junction is an extremely low operation temperature in the lower-millikelvin region. This is, on the other hand, the advantage of SPS's realized by nitrogen-vacancy centers in diamond operating at room temperature. ${ }^{4}$ The simplified model of the luminescent defect is a four-level structure with two nonradiating and one photon emitting transition. After laser excitation antibunched photons are emitted.

Besides the realizations of SPS's by the use of selforganized InAs quantum dots in optically excited ${ }^{5}$ and electrically pumped structures ${ }^{6}$ also one realization of a SPS by the use of surface acoustic waves (SAW's) has been proposed. ${ }^{7}$ The basic idea of this device is the use of a single-electron transport mediated by the SAW's towards a lateral $p-n$ junction. The superposition of the piezoelectric
SAW field and the potential of the one-dimensional channel leads to the creation of dynamic quantum dots (QD's) in which single electrons can be transported through the channel. If these electrons are transported towards a lateral $p$-type region, they can recombine with the existing holes and single photons can be emitted. Even though no working device with single electrons has been realized so far, ${ }^{8}$ this idea represents the realization of a SPS.

In this paper, we report on experimental progress on realizing a different approach towards a periodically pumped SPS mediated by a SAW. ${ }^{9}$ The basic mechanism is sketched in Fig. 1. Electron-hole pairs are excited by laser radiation in an empty quantum well [(a) in Fig. 1]. The lateral piezoelectric potential of a SAW dissociates the excitons and also leads to a periodic modulation of the semiconductor band structure. Therefore the electrons relax to the local minima of the conduction band and the holes relax to the local maxima of the valence band [(b) in Fig. 1]. The resulting decrease in wave function overlap increases the lifetime of the electrons and holes drastically. Then the charges are transported laterally in the semiconductor structure by the propagating SAW $[(\mathrm{c})]$. If a sufficiently deep single QD potential is superimposed to the band structure, one single electron and one single hole relax successively into the QD potential per wave cycle $[(d)+(e)$ in Fig. 1], resulting in a determining emission

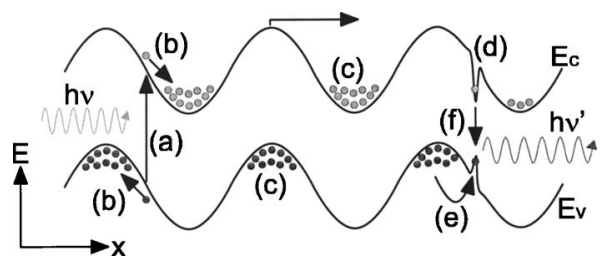

FIG. 1. A simpified schematic picture of a single-photon escalator: laser-generated electron-hole pairs are dissociated by a surface acoustic wave and transported towards a single quantum dot where they recombine and single photons are emitted. 


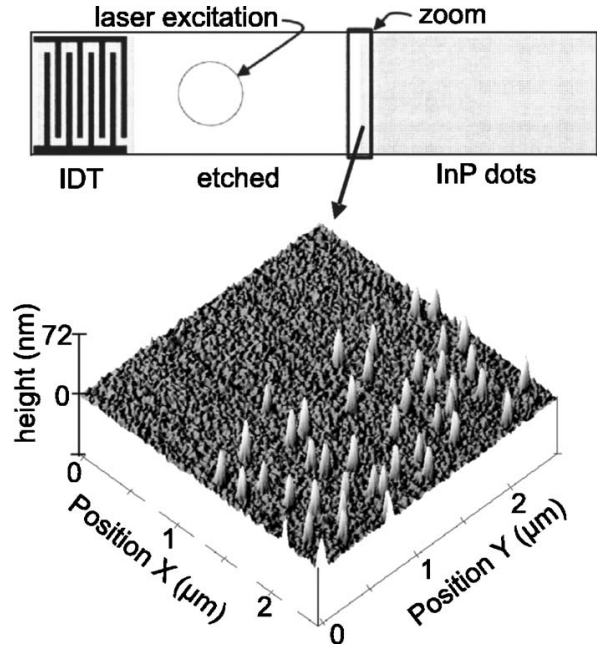

FIG. 2. Sample $A$ : GaAs/InGaAs heterostructure with $\mathrm{InP}$ islands at the surface. In most parts of the sample the InP islands have been removed chemically and an interdigital transducer to launch a SAW has been processed. At the bottom an AFM picture of the etched-unetched transition is shown.

of single photons with a repetition rate given by the SAW frequency.

Measurements of two almost identical samples are presented in this paper. Based on a GaAs substrate first a 125-nm-thick GaAs buffer and then a 7-nm $\operatorname{In}_{0.2} \mathrm{Ga}_{0.8} \mathrm{As}$ quantum well are grown by metal organic chemical vapor phase epitaxy followed by an undoped GaAs cap layer of $20 \mathrm{~nm}$ thickness, leaving the quantum well empty of charge carriers. Finally, at the surface of the sample self-organized $\mathrm{InP}$ islands are grown in the Stranski-Krastranov growth mode $^{10,11}$ ( 3 monolayers). The islands have an average diameter of $80 \mathrm{~nm}$, a height of $22 \mathrm{~nm}$, and a density of 1-2 $\times 10^{9} \mathrm{~cm}^{-2}$ (see close-up part of Fig. 2). Due to a $37 \%$ lattice mismatch of InP and GaAs, the local strain underneath the self-organized islands induces potential cavities in the InGaAs quantum well. These stressor-induced quantum dots (STIQD's) are deliberately made for emitting single photons.

In Fig. 2 the layout of sample $A$ is shown. In most parts of the surface the InP islands have been removed chemically using $10 \% \mathrm{HCl}$ for $60 \mathrm{~s}$, leaving the undoped $\mathrm{GaAs}$ layer at the surface. Then an interdigital transducer (IDT) with a period of $5 \mu \mathrm{m}$ has been processed $(5 \mathrm{~nm} \mathrm{NiCr}, 100 \mathrm{~nm} \mathrm{Al}$, $5 \mathrm{~nm} \mathrm{NiCr}$ ) on the etched surface and with a given wave velocity of $v \approx 2865 \mathrm{~m} / \mathrm{s}$ on GaAs a SAW with a frequency of $580 \mathrm{MHz}$ can be launched. A laser diode with a wavelength of $680 \mathrm{~nm}$ is used to create two-dimensional electronhole pairs in the InGaAs quantum well in the area between the IDT and InP islands.

First a nitrogen-cooled charge-coupled device (CCD) has been used to perform energy-resolved measurements in order to characterize the sample without a SAW present. Two single measurements of the photoluminescence (PL) intensity of sample $A$ are presented in Fig. 3. First the part of sample $A$ with the InP islands has been investigated [Fig. $3(\mathrm{a})$. Here the most prominent PL intensity peak is at $1.39 \mathrm{eV}$ which corresponds to the ground state of the stressor-induced quantum dots [see Fig. 1(d)]. Beside this

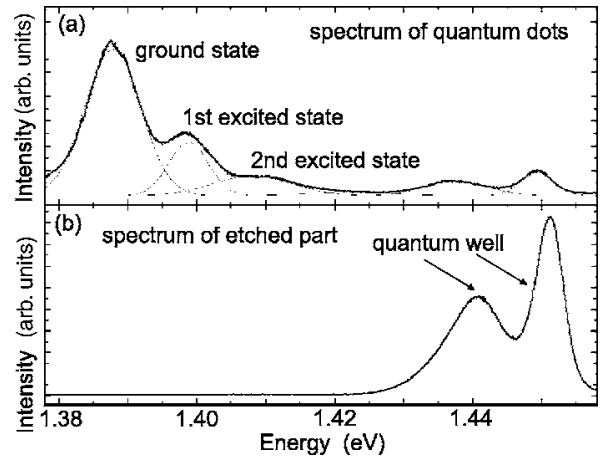

FIG. 3. Photoluminescence spectra of sample A: (a) PL spectrum of the GaAs/InAs heterostructure with InP islands at the surface showing dominating peaks from the induced quantum dots. (b) Spectrum from a part of the sample where the islands have been chemically removed, exhibiting the remaining quantum well peaks.

most dominant peak also the first and second excited states of the quantum dots and weakly the PL of the quantum well are visible. In Fig. 3(b) a spectrum of the etched region of sample $A$ is shown. In contrast to Fig. 3(a) here the most dominant PL peaks can be allocated to the quantum well whereas almost no signal from the stress-induced QD's can be detected. In Fig. 4 a whole series of the PL intensity measurements is presented as a gray scale coded plot as a function of the lateral position on the sample and photon energy. A schematic drawing of the sample layout is added on the right side for clarity. In the area where the InP QD's have been chemically removed $(x=[0,800 \mu \mathrm{m}])$ a signal at $E \approx 1.45 \mathrm{eV}$ has been detected resulting from recombination of electrons and holes in the InGaAs quantum well, whereas in the area with InP QD's (between $x=800 \mu \mathrm{m}$ and $x=1500 \mu \mathrm{m}]$ ) the main PL signal is shifted towards a lower energy of $E \approx 1.39 \mathrm{eV}$ caused by primary recombinations in the STIQD's. The measurements shown in Figs. 3 and 4 are proof that the InP dots can be removed completely by the wet

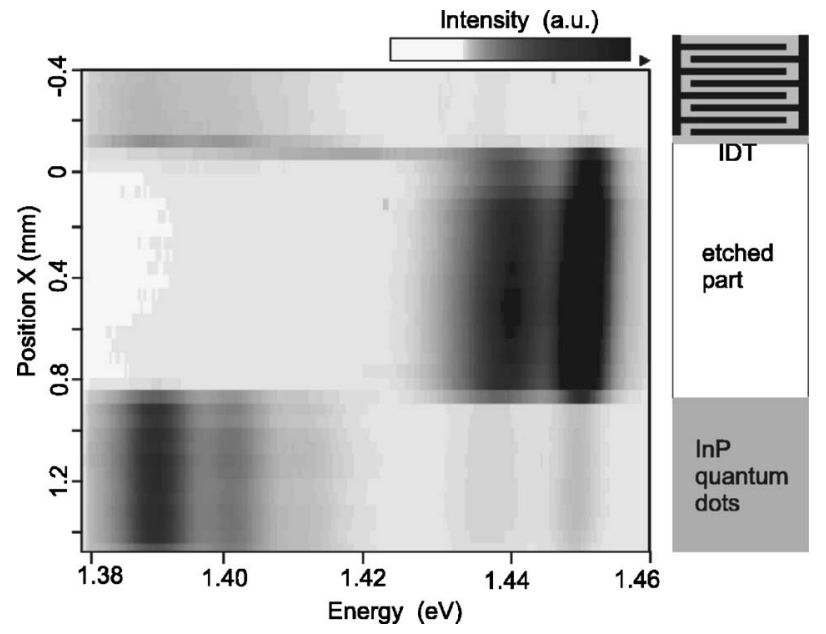

FIG. 4. Gray-scale-coded plot of the PL intensity as a function of lateral position on sample $A$ and photon energy. In the area where the InP QD's have been removed chemically there is a PL signal from the InGaAs quantum well where in the other area the electrons and holes primarily recombine in the InP QD's. 


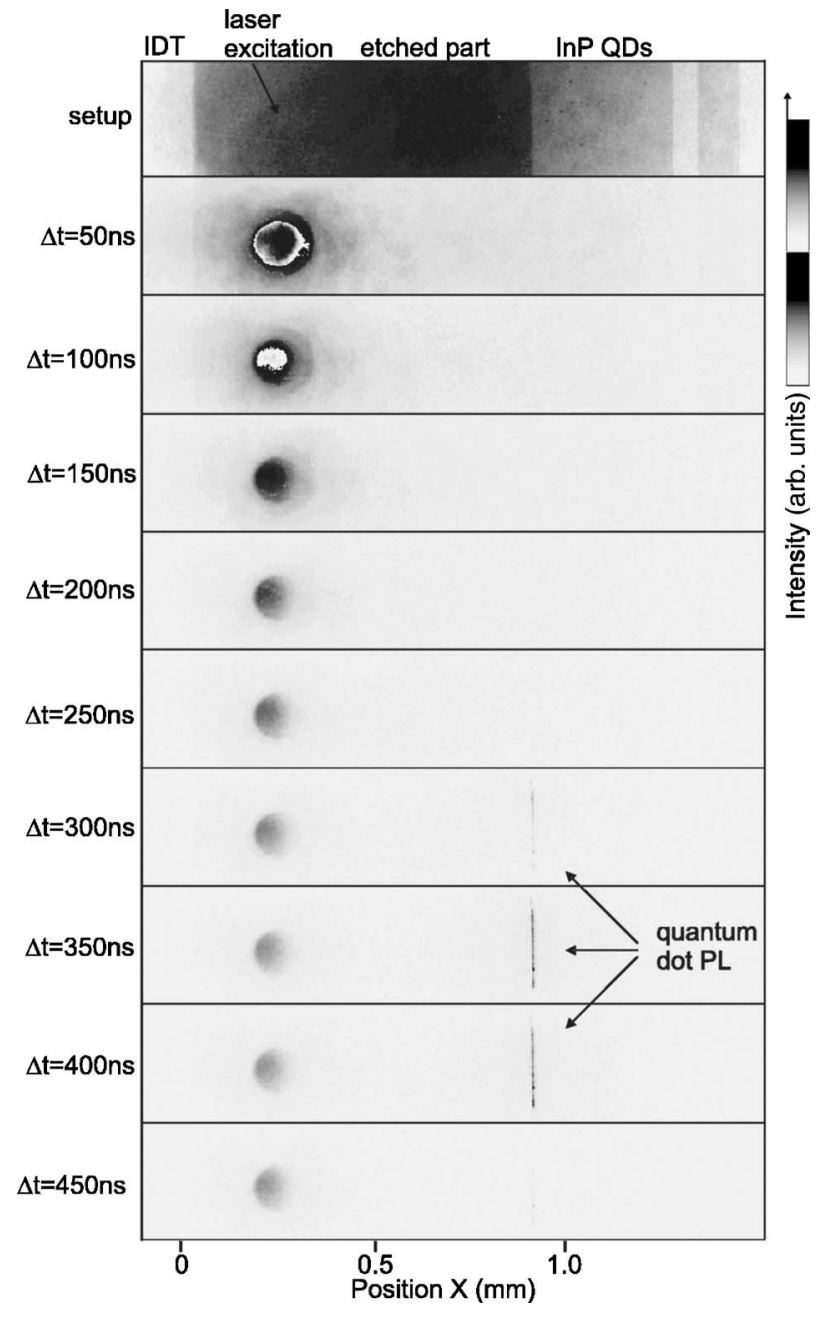

FIG. 5. Lateral and temporal PL signal presentation showing the acousto-optic pumping of QD's (black vertical lines at $t=[300 \mathrm{~ns}, 400 \mathrm{~ns}])$.

etching process without modifying the InGaAs quantum well, causing an abrupt change in emission energy at the etched-nonetched transition.

In the remaining part of the paper measurements are presented where an intensified CCD (4 picos, Stanford Computer Optics) has been used in order to get a better lateral and time resolution. Due to the fact that this measurement setup with the ICCD has no energy resolution, both the PL of the quantum well and of the QD's contribute to the ICCD signal. Therefore the investigations shown in Fig. 4 were necessary. Figure 5 presents a lateral- and time-dependent measurement with the use of a SAW. The top panel shows the direct PL of the sample under continuous illumination and with no SAW present. This panel is used to elucidate the sample geometry in this experiment. The following panels represent the results of a time-resolved experiment with a SAW present on the sample. A SAW is launched on the left side of the sample (at $t=0 \mathrm{~s}$ ), and next to it in the etched area the laser diode is used in a pulsed mode to excite electron-hole pairs. The signal from direct recombination processes of surface states can clearly be seen in the first frame for $t=50 \mathrm{~ns}$ at the spot where the laser was focused to. To improve contrast, the

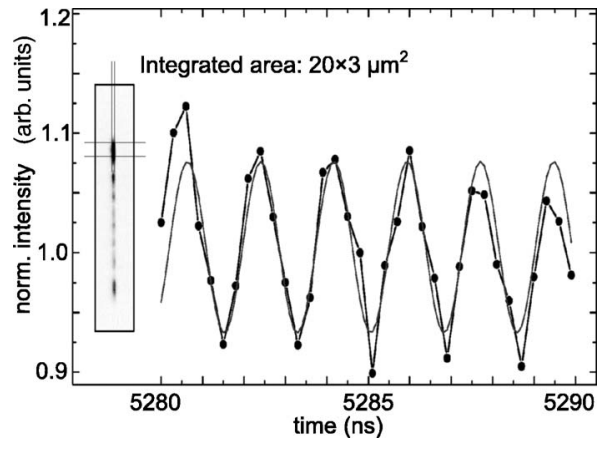

FIG. 6. Temporal intensity evolution integrated over an area of $20 \times 3 \mu \mathrm{m}^{2}$. An oscillation of the signal with a periodicity given by the SAW frequency is visible.

intensity range is twice from white (low intensity) to black (high intensity). The laser excited surface states have a long lifetime which can be seen in Fig. 5 because there is still a PL signal identifiable at the excitation site for $\Delta t=450 \mathrm{~ns}$. As described in Fig. 1 the SAW potential dissociates the lasergenerated electron-hole pairs and transports them laterally towards the STIQD's. This mechanism emerges at the time interval $\Delta t=[300 \mathrm{~ns}, 400 \mathrm{~ns}]$ and can be seen in Fig. 5 as a small vertical black line at the etched-nonetched transition. This time period of $100 \mathrm{~ns}$ of PL from the QD's corresponds well with the SAW pulse duration of $100 \mathrm{~ns}$. Also the time delay of $300 \mathrm{~ns}$ conforms with the time a SAW needs to propagate the $900 \mu \mathrm{m}$ towards the InP QD's $(v \approx 2865 \mathrm{~m} / \mathrm{s})$.

In order to gain more information about the microscopic processes leading to this photon emission from the STIQD's, a PL intensity evolution with high temporal resolution has been performed. In Fig. 6, the normalized PL intensity is shown as a function of time for a certain area of sample $A$. This region is highlighted as an inset and consists of a $20 \times 3 \mu \mathrm{m}^{2}$ area directly after the transition etched part and InP islands. Taken into account a density of $2 \times 10^{9}$ islands per $\mathrm{cm}^{2}$, Fig. 6 represents the averaged PL intensity of 1200 STIQD's. The ICCD signal exhibits an oscillation of $\pm 10 \%$ with a period of $1.8 \mathrm{~ns}$, meaning a frequency of $f=566 \mathrm{MHz}$, corresponding well with the SAW frequency of $f_{S A W}=580 \mathrm{MHz}$. These periodic changes in PL intensity provides the first hint that the photon emission is intentionally with a frequency defined by the SAW frequency.

An ideal single-photon source would consist of one single emitting QD. As a first attempt towards this final goal a second sample has been processed where the interdigital transducer has been replaced by a focusing one in order to minimize the number of emitting STIQD's. Focusing IDT's are a special form of transducers that are able to focus the emitted SAW onto a small area. First layouts with spherically shaped IDT's have already shown resonable performance ${ }^{12}$ but it has been shown ${ }^{13}$ that a parabolic layout taking the slowness curve of the substrate into account increases the performance to focus the power towards one single spot. Due to limitations of producing curved geometries by the used $e$-beam lithography setup, the ideal layout had to be approximated by ten linear segments as shown in Fig. 7. Sample $B$ only deviates from the layout of sample $A$ by the different shape of the IDT. 


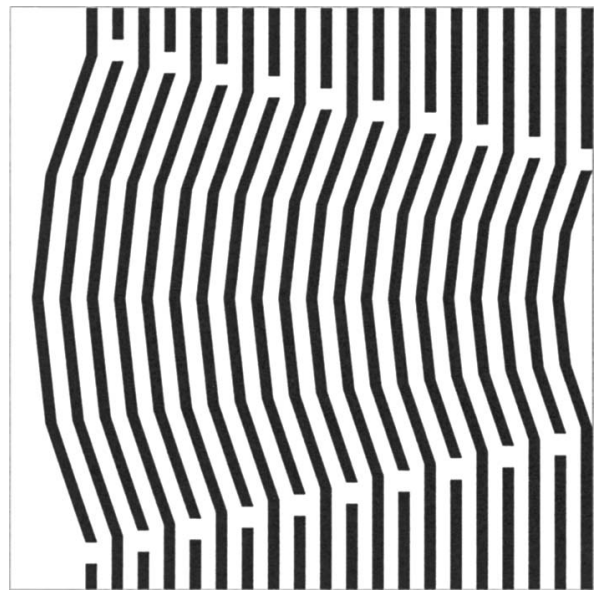

FIG. 7. Layout of the processed interdigital transducer on sample $B$ where a focusing SAW can be launched with.

A picture of sample $B$ is presented at the top panel of Fig. 8 where the parabolic shape of the IDT is clearly visible. For clarity, also the SAW path and the region with the remaining InP QD's are highlighted. In Fig. 8, a similar temporal evolution (here with sample $B$ ) as presented in Fig. 5 is shown. For the complete time series between $\Delta t=[100 \mathrm{~ns}, 500 \mathrm{~ns}]$

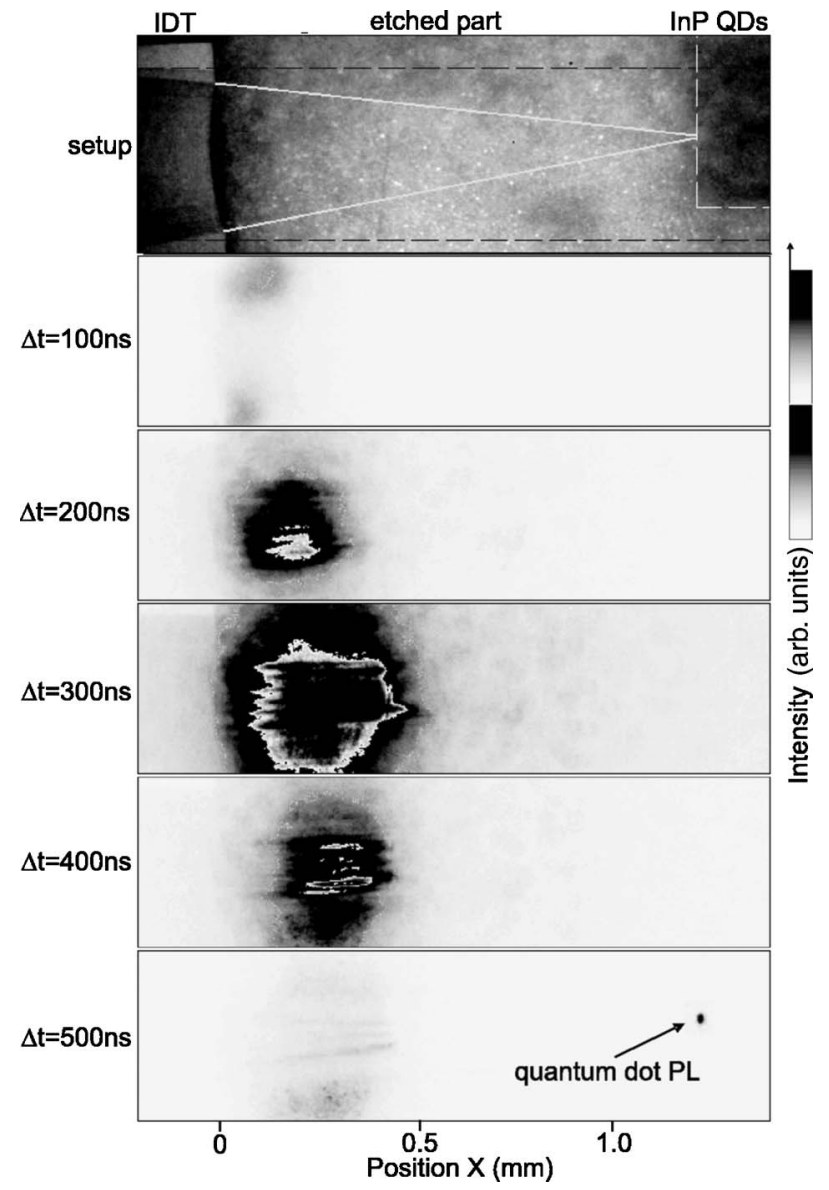

FIG. 8. Lateral and temporal PL signal presentation showing the acousto-optic pumping of a small number of QD's using sample $B$. A picture of this sample is added at the top for assistance.

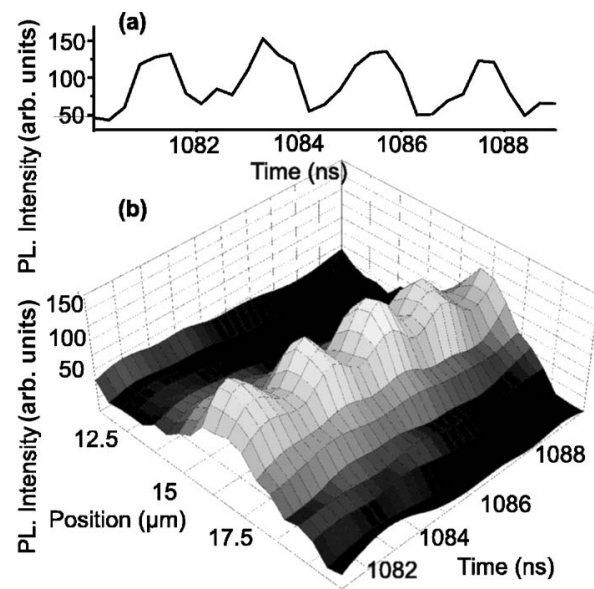

FIG. 9. Temporal intensity evolution integrated over a distance of $2 \mu \mathrm{m}$. An enhanced beating of the signal with a periodicity given by the SAW frequency is visible.

an ICCD signal is visible next to the focusing IDT where the sample has been illuminated by a short laser pulse. Again, this signal has its origin in recombination processes of laserexcited surface states. But in contrast to the measurements presented in Fig. 5 where a vertical line of PL signal has been detected here in Fig. 8 just one single spot of PL signal has been measured for $\Delta t=500 \mathrm{~ns}$. Again, the dissociated electrons and holes are transported by the SAW towards the STIQD's but with sample $B$ the region of recombination has been decreased significantly by the use of the focusing IDT's.

In Fig. 9 a detailed time evolution of this single spot is presented. For these measurements the lateral resolution has been increased, resulting in a focus of $\approx 2 \times 2.5 \mu \mathrm{m}^{2}$, meaning an averaged signal over only $\approx 100$ STIQD's. Now, the ICCD signal exhibits a clear oscillation of $\pm 30 \%$ with a frequency of $f=480 \mathrm{MHz}$, which corresponds within experimental uncertainties with the SAW frequency of sample $B$ of $f_{S A W}=508 \mathrm{MHz}$. The exciton lifetime in semiconductor quantum dots has been found to be in the order of $\tau=1.3 \mathrm{~ns},{ }^{14,15}$ and by using a resonant cavity it can be reduced to $\tau \approx 0.3 \mathrm{~ns}$, which is lower than the repetition frequency given by the SAW, $1 / f \approx 2 \mathrm{~ns}$, ensuring the emission of single photons.

In summary, we have performed experiments of acoustooptic photon emission. The laser-generated electron-hole pairs were dissociated by the piezoelectric SAW potential and laterally transported towards stressor-induced QD's in an InGaAs quantum well. By the use of a focusing IDT we were able to reduce the number of emitting QD's down to $\approx 100$ dots. By further selection processes-e.g., e-beam lithographically defined wet-etching techniques-it can be envisaged that the number of participating QD's can be reduced to 1. Then a periodically pumped single-photon source would have been realized.

This work has been partially funded by the Deutsche Forschungsgemeinschaft DFG (SFB 348). The authors like to thank F. Haake, A. O. Govorov, and J. P. Kotthaus for valuable discussions. 
${ }^{1}$ D. Bouwmeester, A. Ekert, and A. Zeilinger, The Physics of Quatum Information (Springer, Berlin, 2000).

${ }^{2}$ W. Tittel, G. Ribordy, and N. Gisin, Phys. World 11, 41 (1998).

${ }^{3}$ J. Kim, O. Benson, H. Kan, and Y. Yamamoto, Nature (London) 397, 500 (1999).

${ }^{4}$ C. Kurtsiefer, S. Mayer, P. Zarda, and H. Weinfurter, Phys. Rev. Lett. 85, 290 (2000).

${ }^{5}$ P. Michler, A. Imamoglu, M. D. Mason, P. J. Carson, G. F. Strouse, and S. K. Buratto, Nature (London) 406, 968 (2000).

${ }^{6}$ Z. Yuan, B. E. Kardynal, R. M. Stevenson, A. J. Shields, C. J. Lobo, K. Cooper, N. S. Beattie, D. A. Ritchie, and M. Pepper, Science 295, 102 (2002).

${ }^{7}$ C. L. Foden, V. I. Talyanskii, G. J. Milburn, M. L. Leadbeater, and M. Pepper, Phys. Rev. A 62, 011803 (2000).

${ }^{8}$ M. Cecchini, V. Piazza, F. Beltram, D. G. Gevaux, M. B. Ward, A. J. Shields, H. E. Beere, and D. A. Ritchie, Appl. Phys. Lett.
86, 241107 (2005).

${ }^{9}$ C. Wiele, F. Haake, C. Rocke, and A. Wixforth, Phys. Rev. A 58, R2680 (1998).

${ }^{10}$ M. Sopanen, H. Lipsanen, and J. Ahopelto, Appl. Phys. Lett. 66, 2364 (1995)

${ }^{11}$ H. Lipsanen, M. Sopanen, and J. Ahopelto, Phys. Rev. B 51, 13868 (1995).

${ }^{12}$ W. Sauer, M. Streibl, T. H. Metzger, A. G. C. Haubrich, S. Manus, A. Wixforth, J. Peisl, A. Mazuelas, J. Härtwig, and J. Baruchel, Appl. Phys. Lett. 75, 1709 (1999).

${ }^{13} \mathrm{~F}$. W. Beil, private communications.

${ }^{14}$ R. M. Thompson, R. M. Stevenson, A. J. Shields, I. Farrer, C. J. Lobo, D. A. Ritchie, M. L. Leadbeater, and M. Pepper, Phys. Rev. B 64, 201302(R) (2001).

${ }^{15}$ G. S. Solomon, M. Pelton, and Y. Yamamoto, Phys. Rev. Lett. 86, 3903 (2001). 